\title{
Study Element
}

National Cancer Institute

\section{Source}

National Cancer Institute. Study Element. NCI Thesaurus. Code C105752.

An artifact that is one of the individual components of the study. 